\title{
Philosophiques
}

\section{Les récurrences du platonisme chez Descartes}

\section{Josiane Boulad-Ayoub}

Volume 23, numéro 2, automne 1996

URI : https://id.erudit.org/iderudit/027406ar

DOI : https://doi.org/10.7202/027406ar

Aller au sommaire du numéro

Éditeur(s)

Société de philosophie du Québec

ISSN

0316-2923 (imprimé)

1492-1391 (numérique)

Découvrir la revue

Citer cet article

Boulad-Ayoub, J. (1996). Les récurrences du platonisme chez Descartes.

Philosophiques, 23(2), 405-415. https://doi.org/10.7202/027406ar d'utilisation que vous pouvez consulter en ligne.

https://apropos.erudit.org/fr/usagers/politique-dutilisation/ 


\title{
IES RÉCURRENCES DU PLATONISME CHEZ DESCARIES
}

\author{
PAR \\ Josiane BOULAD-AYOUB
}

Descartes a affirmé lui-mème, à maintes reprises, sa rupture
d'avec le passé philosophique ou scientifique. L'histoire a sanctionné
ces affirmations puisqu'on a coutume de faire débuter par la pensée cartésienne, la première transformation profonde du discours philosophique. Certaines récurrences de la philosophie platonicienne ne laissent pas, néanmoins, d'ètre repérables chez Descartes. Si ces infiltrations ne sont pas très nombreuses, elles peuvent, cependant, être mises en évidence sur au moins trois exemples: i) le rôle du modèle mathématique, ii) la méthode, iii) la thèse de l'innéisme des idées. Tous trois mettent en jeu les procédés, les concepts, les thèses que propose Descartes pour atteindre la connaissance. Ainsi la métaphysique cartésienne prend son essor à partir de la méthode pour poser les fondements de la révolution de pensée qu'elle opère au $\mathrm{XVII}^{e}$ siècle mais c'est le modèle mathématique qui permet de constituer la méthode de façon opératoire, cette méthode dont l'agencement règle les structures des deux premières Méditations, par exemple. L'évidence du mathématicien offre le type d'une certitude qui se renouvelle chaque fois que s'impliquent essence et existence ; c'est le cas pour le cogito, c'est le cas pour l'idée de Dieu. À son tour la méthode permet l'application généralisée du modèle qui l'a fécondée ; elle autorise une reconstruction de la mathématique et de toute la science cartésienne.

Faute de temps, je me limiterai ici au seul examen de l'innéisme pour relever les marques du platonisme dans la métaphysique cartésienne. Un certain platonisme sera ainsi décelable chez Descartes qui met l'accent sur l'âme mais n'oublie pas quelque chose d'essentiel, non pas de l'idée, mais en rapport avec l'idée : si l'idée est du monde divin, nous avons comme une nostalgie en nous de ce monde divin. Le philosophe moderne refuse de se placer d'emblée dans le monde des Formes; il commence par montrer la distance entre nos idées et Dieu (théorie de la création des vérités éternelles) mais il lui reste comme une inquiétude, et de l'Idée au sens platonicien et du Bien au sens platonicien : l'àme sera elle-même l'inquiètude de l'idée.

Les réserves à faire en parlant du platonisme de Descartes tiendront, comme je le montrerai, en conclusion, aux différences entre l'exemplarité des Formes platoniciennes et l'actualité des idées cartésiennes. L'idée, chez Descartes, est indice et moyen de connaissance non pas tant parce qu'elle est exemplaire, ni mème parce qu'elle est faite sur le modèle divin; elle est elle-mème connaissance actuelle : avoir l'idée de Dieu, c'est actuellement penser à Dieu. Entre l'exemplarité de Platon et l'actualité de Descartes, il y a la même différence qu'entre le statut ontologique de l'idée chez l'un, et 
son statut " psychologique "qui détermine sa fonction épistémologique chez l'autre. Chez Platon, c'est l'Idée qui est au-dessus de tout et qui explique tout, tandis que ce rapport est renversé chez Descartes pour qui c'est surtout l'âme qui compte, le moi qui compte.

\section{Inneisme des idées}

Dans les Regulæe, Descartes fait une rëférence, sans mentionner Platon, à " ces créateurs de la philosophie" qui ne voulaient " admettre à l'étude de la sagesse personne qui füt ignorant de la mathématique ". Nous savons l'affinité chez Platon entre les objets mathématiques et les objets intelligibles dits Idées. Or Descartes, à propos des objets mathématiques les appelle dans les Méditations autant que dans les Regulæ des "natures vraies et immuables" ": cette désignation s'appliquerait parfaitement aux Idées telles que conçues par Platon. Chez ce dernier les Idées sont de "vraies et immuables natures"; les objets mathématiques sont des essences immuables et éternelles qui s'imposent à la pensée avec un caractère de nécessité . $^{3}$.

Comment connaitre ces "vraies et immuables natures "? L'un des procédés premiers pour la connaissance de la réalité intelligible est, chez Platon, la réminiscence ${ }^{4}$ qui est elle-mème un aspect de la mèthode platonicienne : à partir des exemples mathématiques, arriver à la connaissance et finalement à l'Idée. La doctrine de la réminiscence assure ainsi dans la théorie de la connaissance platonicienne une double fonction : épistémologique, car elle implique l'exigence de principes a priori et la notion que le monde sensible est la copie du monde intelligible ; ontologique, car la réalité du monde des idées rend possible le savoir. La confrontation de cette doctrine avec la thèse cartésienne de l'innéisme qui en est comme une transposition, permet de determiner, en ce qui concerne la théorie des idées, des effets analogues à ceux de la doctrine platonicienne, ceci sous le même double aspect, épistémologique et ontologique.

Rappelons brièvement ce qui chez Descartes est posé comme notions innées, naturelles ou primitives : ce sont les idées vraies ; l'homme les découvre en son esprit: "elles sont toutes mentibus nostris ingenitæ $e^{5}$ ". La règle IV des Regulæ affirme que l'intelligence humaine "a je ne sais quoi de divin "[où] "les premières semences des pensées utiles ont été déposées ${ }^{6}$ ". Ces idées vraies, celles de Dieu, de l'esprit, du corps, du triangle, c'est-à-dire toute idée qui représente, selon Descartes, les essences vraies, immuables,

1. Descartes, Règle IV, Règles, A.T., X, 375.

2. Descartes, A.T., IX, 51, par exemple.

3. Cf. Platon, Ménon; Phèdre 247e-249b ; Phédon 65a ; 88a-b.

4. La doctrine de la réminiscence est exposée dans le Ménon, $82 \mathrm{a}$ ss, le Phédon 74e ss ; voir également les allusions du Phèdre $240 \mathrm{c}$ et du Théétète $149 \mathrm{a}-150 \mathrm{~cd}$.

5. Descartes, à Mersenne, 15 avril 1630, A.T., I, 145.

6. Descartes, Règles, A.T., X, 373. 
éternelles ${ }^{7}$ renvoient, en fait, à toutes les idées per se notæ qui n'enveloppent aucune affirmation ni négation ${ }^{8}$.

Il faut cependant différencier le rôle des idées innées : l'idée de Dieu n'est pas reflet des objets extérieurs, ses attributs sont concevables, non imaginables; l'idée d'âme, elle, est condition de toute autre pensée puisque "c'est par elle que nous concevons toutes choses"; aussi se voit-elle définie comme "une chose capable de penser à tout ce que nous pensons ${ }^{9}$ ". Les notions du nombre, de la durée qui concernent les liaisons sont mixtes; c'est pourquoi la Règle XII y adjoint les axiomes, "notions mixtes qui sont comme des liens destinés à unir entre elles d'autres notions simples et sur l'évidence desquelles repose la conclusion de tout raisonnement ${ }^{10}$ " Ces notions simples éclaireront l'esprit dans l'élaboration d'une physique scientifique ; la theorie s'inspire des affirmations de l'Antiquité, en particulier du Théétète, sur les éléments derniers irréductibles à l'analyse, éléments simples ou atomes du monde. Quand les éléments simples se mélangent, ils forment des notions mixtes susceptibles d'explication; une fois les éléments simples connus, les éléments mixtes peuvent l'être aussi à condition de retracer la façon dont s'est produite la concaténation des éléments simples en éléments complexes.

Les Remarques de Descartes sur le placard de Regius constituent finalement l'exposé le plus complet sur la nature de l'innéisme cartésien. Descartes y remarque que l'innéité est un pouvoir de l'esprit et désigne les critères pour reconnaitre ce genre d'idées : elles ne "procèdent ni des objets du dehors ni de la détermination de ma volonté mais seulement de la faculté que j'ai de penser ${ }^{11}$ ". Mon esprit forme de façon innée non seulement les idées de Dieu, de l'âme, du mouvement mais même les idées de la douleur, des couleurs, des sons et de toutes les choses semblables ${ }^{12}$, car l'esprit se représente ainsi les mouvements corporels qui en sont l'occasion. Toutes ces idées sont toujours en nous "en puissance "

Regius a été incapable de voir le mouvement général des Méditations qui vise à éloigner l'homme des sens pour faire repartir toutes nos vérités de l'esprit et de ses idées, ce mouvement qui va des concepts aux choses. La notion d'inneité consiste alors en la faculté de produire les idées, de penser. Descartes marque par là l'indépendance de la pensée vis-à-vis de l'indépendance de l'étendue $^{14}$. Les idées innées ne sont pas des connaissances actuelles imprimées en nous dès le sein de notre mère, elles ne sont pas perpétuellement sous le regard de notre lumière naturelle, leur

7. Descartes, à Mersenne, 16 juin 1641, A.T., III, 383.

8. Du même au même. 22 juillet 1641 . A.T., III, 418.

9. Descartes, à Mersenne, 16 juin 1641, A.T., III, 383.

10. Descartes, Règles, A.T., X, 419.

11. Descartes, Notæe in programma, A.T., VIII, $2^{2}$ partie. 358.

12. Descartes, ibid., A.T., VIII. $2^{e}$ partie, 359 ; en ce sens l'idée des couleurs serait innée chez l'aveugle, mais il ne peut l'utiliser : cf. Ve Objec. et Rép.. A.T., III, 363.

13. Descartes, ibid. : A.T., VIII, $2^{\mathrm{e}}$ partie, 361.

14. Descartes, Principes, A.T., VIII, 357. 
innéité se réduit au pouvoir que nous avons de les produire, remarque Descartes à Hobbes ${ }^{15}$.

\section{Fonction ontologique}

Comment tirons-nous parti de ces notions innées? Le platonisme doit expliquer pourquoi l'esprit humain naturellement en possession des ètres intelligibles ne les considère pas immédiatement de façon claire. La doctrine de la réminiscence l'explique en faisant porter sur le corps l'obstacle qui se place entre notre faculté de connaitre et l'objet de notre connaissance. Descartes, dans l'Épître à Voët. fait allusion à Socrate qui, interrogeant un enfant, lui fait redécouvrir des vérités qu'il tire de son propre esprit ${ }^{16}$ et prouve ainsi, dit-il, sa théorie de la réminiscence. Descartes s'inspire de cet exemple pour ajouter que nous connaissons non par l'expérience de nos sens mais par les forces de notre propre intelligence : c'est ainsi que nous avons la connaissance de Dieu en tirant des vérités de notre propre esprit $^{17}$. Or quelles sont les vérités fondamentales que Descartes tient à prouver ? Ce sont le cogito, l'existence de Dieu, la distinction entre l'âme et le corps, autrement dit, les idées de Dieu, de l'esprit, de l'étendue...

La thèse de l'innéisme sera la pièce nécessaire pour fournir les fondements de ces démonstrations, sans sortir de l'âme seule et sans faire intervenir le corps. Pour cela il fallait revenir au platonisme et à l'augustinisme et abandonner la philosophie régnante de Saint Thomas. Gilson a montré comment tout un courant d'origine platonicienne se "trouve pendant le Moyen-Áge et au début du XVI siècle [...] qui annonce l'augustinisme de l'Oratoire tel qu'il se manifestera chez Malebranche ${ }^{18}$. La doctrine des idêes innées est reçue sans discussion par Descartes. Il ne l'invente pas : elle a cours dans le milieu philosophique et théologique fréquenté par lui dès les années 1628. Il utilise et adapte à ses besoins cette doctrine d'origine platonicienne qu'il trouve propagée dans son milieu. Chez Platon la réminiscence est surtout un procédé, une méthode pour démontrer que la réalité se trouve établie a priori et qu'il faut remonter à ces a priori si l'on veut appréhender la réalité et connaitre. Chez Descartes, certaines notions sont posées comme premières, primitives : elles sont la condition de notre connaissance et nous partons d'elles pour pouvoir déduire les autres vérités ; elles constituent les principes généraux d'explication sur lesquels reposeront les démonstrations. C'est l'ensemble du monde des Idées, entités supra-humaines qui, chez Platon, correspondent aux notions primitives cartésiennes. L'analyse du Cogito nous livre ces notions primitives ; c'est dire que ces notions sont innées mais qu'elles se situent dans l'esprit humain. La portée ontologique de l'innéisme est comparable à celle de la réminiscence : la réalité des idées rend possible la connaissance.

15. Descartes, III Obj. et Rép., A.T., LX, 147.

16. Descartes, Épütre à Vœetius, A.T., VIII, II, 167.

17. Descartes, ibid.

18. E. Gilson, Études sur le rōle de la pensée médiévale dans la formation du système cartésien, Paris, Vrin, 1967, p. 29. 
Ainsi chez Descartes les preuves de l'existence de Dieu fondées sur le seul contenu d'une pensée distincte de l'étendue sont permises par l'innéisme. L'idée de Dieu est une idée innée ; c'est elle qui est le seul point de départ possible de la preuve : le contenu de cette idée ne peut avoir d'autre cause que Dieu ${ }^{19}$. Dans le cartésianisme où du connaître à l'ètre, la conséquence est bonne, on ne peut tirer la réalité de l'idée qu'en s'appuyant sur son innéité et pour pouvoir énoncer la règle constante: "quand nous disons que quelque attribut est contenu dans la nature ou dans le concept d'une chose, c'est de même que si nous dirions que cet attribut est vrai de cette chose, et qu'on peut assurer qu'il est en elle ${ }^{20}$.

L'innéisme supporte également la thèse qu'une réalité corresponde à la pensée vraie, la nécessité interne de la pensée prouvant la réalité de son objet. Ainsi les idées claires et distinctes rejoignent de vraies et immuables natures et, par conséquent, des réalités véritables. La première de toutes les évidences, le Cogito, se pose en critère de l'évidence et communique la sienne à toutes les propositions qui en découlent :

Or entre ces choses, il y en a de si claires et tout ensemble de si simples qu'il nous est impossible de penser à elles que nous ne les croyions être vraies : par exemple, que j'existe lorsque je pense ; que les choses qui ont une fois été faites ne peuvent pas n'avoir point été faites, et autres choses semblables dont il est manifeste que l'on a une parfaite certitude ${ }^{21}$

La garantie de cette évidence se trouvera ètre donnée par la preuve de l'existence de Dieu qui sera aussi la preuve de la véracité de nos idées claires et distinctes ${ }^{22}$.

L'union et la distinction à la fois de l'àme et du corps, pièce finale de la métaphysique cartésienne, est également soutenue par l'innéisme : la distinction de l'âme et du corps se pense, leur union se sent. L'esprit humain part du doute, en sort par l'affirmation du

19. Cf. la lettre de Descartes à Mersenne de juillet 1641 , A.T., III, p. 396 : "la considération d'un tel être nous conduit si aisément à la connaissance de son existence que c'est presque la mème chose de concevoir Dieu et de concevoir qu'il existe "; voir aussi la $V^{E}$ Méditation, A.T., II, 54-55 : " car il n'y a rien en soi de plus clair et de plus manifeste... .

20. Descartes, Rép. aux Sec. Obj., déf. IX, A.T., IX, 125 : "cum quid dicimus..." et ss.

21. Descartes, Rèp. aux Sec. Obj., A.T., IX, 114. Le problème classique du " cercle cartésien " qui se pose ici a été clarifié de façon très satisfaisante d'abord par P. Lachièze-Rey ("Réflexions sur le cercle cartésien " dans Descartes, recueil publié par la Revue Philosophique, F. Alcan, 1937. p. 205-225) qui établit une solution psychologique (distinguer entre l'évidence actuelle et l'évidence inactuelle, la première sorte d'évidence entrainant une certitude suffisante, ramener la nature des propositions à cette première évidence ; quant à la garantie divine elle sera fondée sur l'évidence actuelle de l'existence de Dieu) et une solution métaphysique. Il y a deux sortes d'évidence : l'une concemant l'être de l'idêe, l'autre sa valeur probante relativement à l'objet qui lui correspond : Dieu étant cause et objet de son idée, la première évidence justifie la seconde sans qu'il y ait lieu de parler de cercle vicieux. Ensuite par M. Gueroult, dans Descartes selon l'ordre des raisons, tome I, Paris, Aubier 1968, p. 237 à 247, qui précise la véritable nature du problème du cercle et critique les moyens de résoudre le problème : "réduire les deux séries à une seule, soit à celle [relevant del l'absoluité du Cogito soit à celle [relevant de] l'absoluité de Dieu; ou rétablir l'indépendance des deux séries qui ne feraient que s'entrecroiser * .

22. Descartes à Elizabeth, 21 mai 1643, A.T., III, 664 . 
Cogito et finit par en déduire la distinction de laame et du corps. La lettre qu'écrit Descartes à la princesse Élisabeth pour lui expliquer l'union de l'āme et du corps déclare qu'il y a deux choses dans l'âme humaine "desquelles dépend toute la connaissance que nous pouvons avoir de sa nature, l'une desquelles est qu'elle pense, l'autre, qu'étant unie au corps, elle peut agir et pâtir avec lui ${ }^{23}$ "; et pour expliquer l'union Descartes a recours aux notions primitives qui sont en nous "comme des originaux ${ }^{24}$ ". Celles-ci jouent la mème fonction archétypale que chez Platon, celle d'un patron sur lequel nous formons nos autres connaissances. On pourrait à la limite affirmer que le monde n'existe pas puisque entre la substance pensante et la substance étendue n'apparaît pas de liaison nécessaire, mais du méme coup disparaitrait l'union de l'āme et du corps. La pensée ne saurait cependant concevoir qu'elle n'existe pas tandis qu'elle pense, elle trouverait donc toujours en elle ces notions mixtes parmi lesquelles figure le nombre.

\section{Fonction épistémologique}

Venons-en maintenant à la portée épistémologique de l'innéisme. Platon considère que les principes a priori constituent le monde intelligible et que le monde sensible est la copie du monde intelligible. L'innéisme des notions signifie, de mème, pour Descartes, l'antériorité de ces notions, leur a priorisme. Le vocabulaire cartésien lui-mème se fait platonicien pour parler de cet apriorisme et présente jusqu'à des concordances verbales: ainsi le reminisci de la Cinquième Méditation, les termes de mélange, participation dans les Règles ${ }^{25}$, l'affirmation de l'article 18 de la Première partie des Principes : "il est impossible que nous ayons l'idée ou l'image de quoi que ce soit, s'il n'y a en nous ou ailleurs un original qui comprenne en effet toutes les perfections ${ }^{26} "$. Dans la Troisième Méditation nous assistons comme à une montée vers l'idée première, vers l'idée de Dieu; le mouvement mème de montée, les termes de "déchoir", de " patron " ${ }^{27}$ ont une résonance bien platonicienne.

Sans doute Descartes appelle idée ce que Platon appelait image ou ombre mais l'analogie existe : le rapport entre ce que Descartes appelle idée et son original ou son objet est le même que celui que Platon conçoit entre l'image et l'idée. Dans la Cinquième Méditation Descartes veut prouver "derechef l'existence de Dieu "; il part alors

23. Descartes, ibid., 665.

24. Descartes, $V$ Méditation, latin, A.T., VII, 64 ; "il ne me semble pas que j'apprenne rien de nouveau mais plutôt que je me ressouviens de ce que je savais déjà auparavant, c'est-à-dire que j'aperçois des choses qui étaient dejà dans mon esprit, quoique je n'eusse pas encore tourné ma pensée vers elle ", A.T., IX, 51.

25. Descartes, Règles... Règle II, A.T., X, 382 ; XII, ibid. p. 422 ; XIV, ibid., p. 438-439.

26. Descartes, Principes... $1^{\text {re }}$ partie, art., I8; A.T., IX, II, 33.

27. Descartes, III Méditation, A.T., IX, 33: " encore qu'il puisse arriver que l'idée donne naissance à une autre idée, cela ne peut pas toutefois ètre à l'infini, mais il faut à la fin parvenir à une première idée, dont la cause soit comme un patron ou un original ", et un peu plus loin, ibid., "la lumière naturelle me fait connaître évidemment que les idées sont en moi comme des tableaux ou des images qui peuvent à la vérité facilement déchoir de la perfection des choses, dont elles ont été tirées * 
des idées mathématiques et expose comment à partir d'une approche attentive des objets mathématiques qu'il nous semblait ignorer, on découvre peu à peu tout ce qui nous semblait caché, c'est comme si on s'en souvenait ${ }^{28}$. Il utilise ici directement ce procédé de la réminiscence pour ou contre ce qui concerne l'idée de Dieu et l'affirmation de la possibilité immédiate de l'existence de Dieu. Aussi montre-t-il que de même que lorsque nous appliquons notre attention à un objet mathématique nous lui découvrons telle ou telle propriété, de même chaque fois que nous pensons avec attention à l'idée que nous avons de Dieu, nous retrouvons Dieu, c'est comme si nous nous en ressouvenions ${ }^{29}$. Descartes ne prend pas à la lettre la réminiscence mais il s'en sert pour exprimer cette antériorité de droit qui n'engage aucune connaissance préalable de la notion. L'idée de l'ètre mathématique est innée, a priori, inhérente à la pensée d'un esprit fini ; elle ne saurait advenir de l'expérience qui est imparfaite alors que l'imparfait n'est qu'une négation du parfait ${ }^{30}$. Bien au contraire, si on veut l'appliquer à tout, elle doit ètre antérieure ả toute expérience sensible.

Descartes répond ainsi à Gassendi qui lui objecte que les figures se forment d'après le modèle aperçu par nos sens ${ }^{31}$ : "Je ne pense pas que jamais aucune partie d'une ligne ait touché nos sens, qui fut véritablement droite ${ }^{32} ;$ si on ne retrouve pas dans le monde une figure comme le triangle qui ait la rigueur de l'essence géométrique, c'est que "l'idée véritable du triangle est déjà en nous " ${ }^{33}$. Gassendi remarque à cet égard que les arguments cartésiens sont des arguments à la Platon : Platonice dicantur (Disquisitio 378b) et fait allusion à ces passages du Phédon (73e-75c) où Platon se réclame de l'Idée de l'Égal en soi et de la réminiscence à la vue d'égalités imparfaites. Cependant cet argument platonicien de Descartes ne nous ramène pas à l'intuition d'un monde intelligible d'idées, l'idée de nombre n'étant "qu'éminemment " et non "formellement " contenue en celle de Dieu $^{34}$ - aussi ne pourrons-nous décider sur l'existence du plus grand nombre ${ }^{35}$; nous ne pourrons tirer l'idée de nombre de

28. Descartes, Véditation, A.T., LX, 51.

29. Descartes, ibid., p. 52 ss.

30. Descartes, II réponses, A.T., VII, 382 et Véponses, p. 162 : cf. aussi dans les Règles, règle II, A.T., X, 364-365.

31. Descartes, $V^{e}$ Objections, contre la V $V^{e}$ Méditation, A.T., VII, 320-321.

32. Descartes, $V^{\mathbb{E}}$ Réponses, ibid., p. $381-382$.

33. Descartes, ibid., p. 382 ; $c f$ dans l'Entretien avec Burman : "je ne pourrais concevoir un triangle imparfait si je n'avais d'abord l'idée du triangle parfait à partir de laquelle je remarque l'imperfection de celui que je vois ".

34. "[...] quaedam in idea Dei formaliter contineri, ut cognitionem et potentiam, alia tantum eminenter, ut numerum et longitudinem * A.T., VII, 137. La définition IV, des $I^{e}$ Réponses, ibid. p. $16 \mathrm{I}$, donnait de même que "les mêmes choses sont dites ètre formellement dans les objets des idées, quand elles sont en eux telles que nous les concevons *, ce qui n'est plus le cas pour êminement. Notons que Malebranche, au contraire, convertira le nombre en archétype éternel.

35. Descartes à Mersenne, mars 1642, A.T., III, p. 544-545: "vous me mandez comme un axiome qui vienne de moi : que tout ce que rous concevons clairement est ou existe; ce qui n'est nullement de moi, mais seulement que tout ce que nous apercevons clairement est vrai, et aussi qu'il existe, si nous apercevons que son existence soit possible. Car bien que l'être objectif de l'idée doive avoir une cause réelle, il n'est pas toujours besoin que cette cause le contienne formaliter mais seulement eminenter *. 
l'idée de Dieu bien que sa cause soit en Dieu lui-mème. Ainsi * le nombre que nous considérons en général sans faire réflexion sur aucune chose créée, n'est point hors de notre pensée, non plus que les autres universaux ${ }^{36}$; il compte parmi les idées que nous tirons de nous-mèmes, avant la preuve de l'existence de Dieu :

[...] quand je pense que je suis maintenant, et que je me ressouviens outre cela d'avoir été autrefois, et que je conçois plusieurs diverses pensées dont je connais le nombre, alors j'acquiers en moi les idées de la durée et du nombre, lesquelles, par après, je puis transférer à toutes les autres choses que je voudrai ${ }^{37}$.

Mème dans l'hypothèse du solipsisme il me suffirait de considérer réflexivement mes pensées et ses opérations pour y trouver lidée du nombre, et Descartes précise par quelle chaine de raisons a priori : "substantia, duratio, ordo, numerus ${ }^{38}$, chaque terme étant le requisit du terme suivant. D'abord substantia, le moi fini et pensant, ayant par cela mème le pouvoir "d'amplifier "ses perfections, par exemple sa connaissance et sa puissance ${ }^{39}$ et la faculté de lier ses idées. Duratio puisque mon imperfection m'engage dans la durée, succession objective et condition de l'énumération ${ }^{40}$. Ordo:l'ordre logique qui me permet dans une succession de distinguer le réel et l'imaginaire ${ }^{41}$. Par ces trois requisits le nombre qui concerne l'entendement pur est possible ${ }^{42}$.

Un problème surgit cependant de savoir si la notion mathematique du nombre est indépendante de l'intuition de l'étendue. Descartes suppose que l'innéité résout ce problème ${ }^{43}$. L'idée d'étendue en ce cas est innèe comme celle du monde ${ }^{44}$. Elle

36. Descartes, Principes, I, paragraphe 58, A.T., IX, 11, 50.

37. Descartes, III Méditation, A.T., IX, 35.

38. Descartes, Principes, $1^{\text {re }}$ partie, parag. 48 ; ibid, paragraphe 55 (durée, ordre, nombrel A.T., VII, p. 43-44, substantia, duratio, numerus, id., A.T., V. P. 335.

39. Descartes, A.T., IX, p. 37.

40. Condillac dira : "Il n'y a point de nombre aux yeux de Dieu. Comme il voit à la fois tout, il ne compte rien. C'est nous qui comptons, parce que nous ne voyons qu'un à un..." Langue des Calculs, chap. IV. Paris 1801, T., I, p. 60. Et Descartes pour Amauld, 4 juin 1648, A T., V, 193 : "dici tamen non posset duratio mentis humanae tota simul, quemadmodum duratio Dei; quia manifeste cognoscitur successio in cogitationibus nostris, qualis in cogitationibus divinis nulla potest admitti...

41. Descartes, à Regius, 24 mai 1640, A.T., III, 66 : " neque video cur velis perceptionem universalium magis ad imaginationem quam ad intellectum pertinere. Ego enim illam soli intellectui tribuo, qui ideam ex se ipsa singularem ad multa refert ".

42. Le texte cité plus haut, Méditation III, A.T., IX, 35, où par * plusieurs diverses pensées dont je connais le nombre . j'acquiers en moi l'idée de nombre, semble le prouver; $c f$. aussi Principes I, paragr. 59, A.T., IX, II, 50, nous universalisons l'idée de deux que nous remarquons en deux pierres : " nous voyons deux pierres et sans penser autrement à ce qui est de leur nature, nous remarquons seulement qu'il $y$ en a deux, nous formons en nous l'idee d'un certain nombre que nous nommons le nombre de deux ", nous faisons de méme pour deux oiseaux, deux arbres, etc.

43. Méme si l'on convient avec J. Laporte, Le rationalisme de Descartes, Paris, PUF, p. $128 \mathrm{ss}$, ou si l'on nie avec M. Gueroult, op. cit., T. I, p. 138, que l'idée d'étendue apparaît avec l'union de l'áme et du corps, cette idée d'étendue appartient bien au groupe des idées innées.

44. Descartes, Méditation III, A.T., IX, 31 . 
n'admet avec elle, en " tant que façon de penser ", " aucune différence ou inégalitét ${ }^{45}$. On introduit néanmoins une inégalité si le nombre concerne d'abord les choses spirituelles, puis les choses matérielles; or l'objet de la mathématique est la substance étendue ${ }^{46}$, non la substance pensante, et l'on conçoit d'autant moins cette inégalité. Descartes, cependant, postulant que les idées des choses qui existent, en tant qu'elles sont en sa pensée, peuvent ètre hors de lui, cite en premier lieu l'étendue, et ajoute : "De plus je puis nombrer en elle plusieurs parties (numero in ea varias partes) ${ }^{47}$ " cela signifie que " quelque partie que nous puissions déterminer len elle] par la pensée doit étre réellement distincte de ses autres parties ${ }^{48}$ ". Ainsi avec l'idée innée de l'étendue, voici que nous avons l'idée innée de parties réellement distinctes - partes extra partes-homogènes, identiques, totalisables, et que Descartes nous présente une notion véritablement mathématique du nombre.

Enfin la thèse de l'innéisme des notions mathématiques permet à Descartes de concevoir l'objectivité scientifique toute entière au delà de la sensation; l'intelligibilité de la science sera indépendante de l'expérience sensible, comme en mathématiques. La subjectivité du sensible résulte de l'union des deux substances; les facultés d'imaginer et de sentir n'appartiennent à l'âme qu'en tant "qu'elle est jointe au corps, en sorte que ce sont des sortes de pensée sans lesquelles on peut concevoir l'āme toute pure ${ }^{49}$ ". En d'autres termes, le subjectif est donc ce qui dans la connaissance du monde s'effacerait de l'àme toute pure, séparée du corps; la confusion sensible résulte de l'intrusion dans l'âme d'un élément non cognitif, effet de la matière. Le voilà exclu de l'inné - d'une façon analogue à celle de Platon puisqu'exclu de la faculté de connaitre. Le voilà exclu également de lanalyse scientifique puisque celle-ci consiste à éliminer du sensible les éléments non cognitifs, c'est-à-dire le sensible lui-même, pour ne garder que les élêments cognitifs qu'il implique : les idées claires et distinctes, relatives à l'étendue ou géométriques. La connaissance véritable déposée dans les idées innées attend d'être retrouvée par nos propres forces intellectuelles: nos déductions développent ensuite ce que "la nature même a gravé et imprimé en nos esprits ${ }^{50}$. Chacun a les moyens de trouver en soimème et par soi-mème ce qui lui est nécessaire pour la connaissance,

45. Descartes énumère : "existentia autem, duratio, magnitude, numeris et universalia omnia... A.T., IV, 349 et dans A.T., VII, 71 : " tum etiam de omni illa natura corporea quae est purae mathesos objectum "; id. VII, p. 384 .

46. Descartes, Méditation V, A.T., VII, 63.

47. Descartes, Principes... I, par. 60 : "unamquamque ejus partes a nobis cogitatione definitam *, A.T., VIII, 28.

48. Descartes au P. Gibieuf, 19 Janvier 1642, A.T., III, 479.

49. Descartes, Lettre au P. Dinet, A.T., VII, 580 ; trad. fr. de Clerselier dans Alquié, II, p. 1088.

50. Rappelons que dans la transposition cartésienne de la thèse platonicienne de la réminiscence en l'innéisme des idées, c'est l'idée de Dieu chez Descartes qui garantit la véracité des idées innées - c'est-à-dire pour lui la faculté de l'esprit humain à produire des pensées - et l'apriorisme des natures mathèmatiques alors que Platon utilise la thèse de la réminiscence pour prouver la réalité des Idées et leur a priorisme sans passer par ce relais de fondement et de justification que constitue l'immutabilité divine. 
l'antériorité des principes rationnels constituant la condition de toute affirmation.

\section{Le déplacement cartésien}

L'hostilité contre la confusion suscitée par le sensible, l'ordre des connaissances réglé par l'ordre des raisons, le prestige des mathématiques et leur rôle pour l'appréhension des structures du monde physique, la méthode en sa fonction libératrice et de modèle générateur de l'appréhension philosophique des vérités fondamentales, la théorie de l'innéisme des idées qui supporte la thèse de l'objectivité de notre connaissance alors qu'elle part d'une subjectivité, autant de traits cartésiens d'un platonisme indiscutable. Descartes, pourtant, nous le rappelions en commençant, ne récuse-til pas lui-mème la plupart de ses rapports avec ses prédécesseurs? Et, en effet, si certains procédés, certains mouvements, certaines théories sont communes à Platon et à Descartes, la visée principale en est radicalement différente. Le sensible pour Platon n'a pas à être connu; nous devons nous élever jusqu'à l'intelligible, seule connaissance, alors que pour Descartes le sensible, s'il n'est pas connaissable immédiatement, peut et doit être connu par la médiation de nos idées. La doctrine cartésienne de l'idée fait dès l'abord apparaître trois ordres de réalité : celui du moi, celui de la chose, celui enfin de l'idée elle-mème qui constitue un plan distinct des deux autres : ici se découvre un monde réel qui sans ètre métaphysique - à l'exception de l'idée de Dieu - est intérieur au cogito et pourtant lui est résistant, un monde où nous retrouverons l'univers de la science sur l'étre duquel a porté le doute de la Première Méditation.

Paradoxalement le point de convergence apparent des deux philosophes, le lieu des idées qui est le lieu de la connaissance, se trouve ètre aussi et, en fait, leur point de divergence : chez l'un, je me détourne du monde sensible, je m'élève jusqu'aux Idées, celles-ci forment un monde intelligible structurant le monde sensible; chez l'autre, je commence aussi par m'éloigner du monde sensible mais ensuite je me retoume vers moi-mème et j'y plonge : je ne sors pas pour ainsi dire de la caverne pour trouver les idées à l'extérieur de moi ; je m'isole en quelque sorte dans la caverne et je m'aperçois que je détiens en moi le pouvoir d'y faire régner la lumière. C'est en mon esprit que je trouve les idées, ce n'est pas en dehors de moi que se trouve la vérité. La vérité demeure à l'intérieur de l'homme, in interiore hominis habitat veritas.

Le pas en avant fait par les philosophes modernes au-delà des découvertes des Anciens est que la connaissance indispensable, source de toute la connaissance, est la connaissance du moi connaissant. Le moi est à luí-même la plus sûre et la plus connaissable de toutes les réalités. Le philosophe moderne ne connait pas seulement son existence mais son essence, sa nature, et il sait par quels procédés il peut progresser vers une connaissance plus poussée. Il sait aussi que la connaissance ne peut pas étre atteinte d'emblée, ni saisie dans son ensemble mais dans une réflexion sans fin. Il découvre en lui cette lumière souveraine qui se 
révèle ètre le "générateur " de sa lumière, celui qui l'éclaire ainsi que le monde qui l'entoure.

Le déplacement introduit par Descartes s'opère sur les idées elles-mêmes et la relation entre l'homme et les idées : pour Platon elles formaient un monde autonome, un réel extra-mental qui ne dépendait de personne : pour Descartes elles sont à la fois essences et tableaux, signes instrumentaux qui, situés en moi, me font découvrir les conditions a priori du monde, et sont autant de rapports entre ma liberté et l'Être puisqu'elles sont assurées par la seule Idée véritablement autonome et créatrice, celle de Dieu.

Département de philosophie

Université du Québec à Montréal 